COMMUNICATIONS IN

ANALYSIS AND GEOMETRY

Volume 11, Number 2, 181-205, 2003

\title{
Mean Curvature Flow of Spacelike Hypersurfaces near Null Initial Data
}

\author{
KLAUS ECKER
}

\begin{abstract}
We prove an interior estimate for the gradient function of spacelike hypersurfaces which move by mean curvature in a Lorentzian manifold. This estimate depends only on a time function which measures how far the hypersurfaces are from being null. As a consequence, we show that under mean curvature flow a weakly spacelike initial hypersurface instantaneously becomes smooth and strictly spacelike except along null geodesics which extend to its boundary.
\end{abstract}

\section{Introduction.}

Let $X_{s}=X(\cdot, s): M^{n} \rightarrow V, s \in\left(0, s_{0}\right)$ be spacelike hypersurface embeddings into a Lorentzian manifold with images $M_{s}=X_{s}\left(M^{n}\right)$. The hypersurfaces $M_{s}$ flow by mean curvature if

$$
\frac{\partial X}{\partial s}(p, s)=H \nu(p, s)
$$

for all $p \in M^{n}$ and $s \in\left(0, s_{0}\right)$ where $H$ and $\nu$ denote the mean curvature and future directed unit normal field of $M_{s}$ respectively.

Spacelike hypersurfaces with special mean curvature function have been an important tool in the study of Lorentzian manifolds. In particular, maximal hypersurfaces, that is hypersurfaces with zero mean curvature were used in the first proof of the positive mass theorem [SY] and foliations of spacetimes by spacelike constant mean curvature hypersurfaces have played an important role in the analysis of the Cauchy problem for the Einstein equations (see [CBY, MT]). We refer to [B3] for an extensive list of references.

Existence and regularity results both for compact hypersurfaces in cosmological spacetimes and for the Dirichlet problem of hypersurfaces spanning a given set of spacelike boundary data were established in [G] and [B1]. In [B1], the existence of maximal hypersurfaces in a class of asymptotically flat spacetimes was proved. All these existence proofs are based on estimates for the gradient function $v=-\langle\nu, T\rangle$ which measures the angle between the 
future directed normal $\nu$ of the hypersurfaces and a fixed future directed timelike vectorfield $T$ on $V$. Such an estimate implies that the quasilinear partial differential equation describing the hypersurface is uniformly elliptic. In [B2], an interior gradient estimate was proved which depends only on a time function measuring how far the hypersurface is from being null. A version of this result in Minkowski space $\mathbf{R}^{n, 1}$ is related to the Cheng-Yau geodesic completeness estimate for constant mean curvature hypersurfaces (see $[\mathrm{CY}]$ and $[\mathrm{B} 2]$ ).

Based on this interior gradient estimate and an intricate time function construction adapted to weakly spacelike hypersurfaces, the boundary value problems were extended to include boundary data with points joined by null geodesics ([B2]). For such boundary data, the spanning hypersurface of prescribed mean curvature contains all null geodesics connecting points on its boundary and is smooth and spacelike away from these 'contained light rays'. This generalizes earlier results of [BS] in Minkowski space.

In [EH1] and [E1], an alternative approach to the existence problem of prescribed mean curvature hypersurfaces was taken via the parabolic equation

$(\mathrm{MCF})_{\mathcal{H}}$

$$
\frac{\partial X}{\partial s}=(H-\mathcal{H}) \nu
$$

for general functions $\mathcal{H} \in C^{\infty}(V)$. This equation has hypersurfaces with mean curvature $\mathcal{H}$ as stationary solutions and includes (MCF) as a special case. As in the elliptic case, results on existence and convergence to stationary solutions rely on an estimate for the gradient function. An additional complication in establishing such an estimate arises from the fact that the mean curvature of the hypersurfaces $M_{s}$ changes and therefore has to be controlled simultaneously. In [EH1], compact hypersurfaces in cosmological spacetimes were considered. In this case, a natural condition on $\mathcal{H}$ and the timelike convergence condition

$$
\overline{\operatorname{Ric}}(Y, Y) \geq 0
$$

for timelike vectorfields $Y$ combined with the maximum principle give control on the mean curvature prior to an estimate on the gradient.

In [E2], noncompact geodesically incomplete solutions of $(\mathrm{MCF})$ in Minkowski space $\mathbf{R}^{n, 1}$ were found which have exponentially growing mean curvature at infinity and in particular do not obey the parabolic maximum principle. To include such solutions in the analysis an interior estimate for solutions of (MCF) in Minkowski space was proved which led to an existence 
result for the Cauchy problem with arbitrary noncompact spacelike initial data.

Theorem 2.1 of this paper provides a version of this estimate in a general Lorentzian manifold which is also a parabolic version of the basic interior gradient estimate in [B2]. In particular, we estimate the mean curvature and the gradient function of the hypersurfaces $M_{s}$ simultaneously. This estimate is independent of the initial values of these quantities. This allows us to solve initial - boundary value problems for compact nonsmooth initial hypersurfaces which contain null geodesics (Theorem 3.2). We prove, analogously to the elliptic case, that the solution of (MCF) agrees with its initial data along the null geodesics which connect boundary points (the singular set ) but is smooth and spacelike away from these curves. Furthermore, the flow converges subsequentially to a hypersurface which is smooth, spacelike and maximal away from the singular set.

All our results extend to solutions of the equation $(\mathrm{MCF})_{\mathcal{H}}$ assuming only a mild condition on $\mathcal{H}$. The timelike convergence condition which we assume throughout this paper can also be relaxed to include the weak energy condition in case the metric on $V$ satisfies the Einstein equations.

This research was supported by the Australian Research Council and the Sonderforschungsbereich 382 at the University of Tübingen, Germany.

\section{Preliminaries and Evolution Equations.}

We consider an $(\mathrm{n}+1)$ - dimensional smooth spacetime $V$ with a Lorentzian metric $\bar{g}$. The metric pairing will be denoted by $\langle\cdot, \cdot\rangle$, the canonical connection by $\bar{\nabla}$ and the curvature tensors by $\overline{\text { Riem }}$ and $\overline{\text { Ric. We shall adopt }}$ the set up and notation of [B2] and assume that $V$ is time oriented and that $T$ is a unit future-directed timelike $C^{2}$ - vectorfield on $V$. We construct a reference Riemannian metric $\bar{g}_{E}$ by

$$
\bar{g}_{E}(Y, Z)=\langle Y, Z\rangle+2\langle Y, T\rangle\langle Z, T\rangle
$$

for vectorfields $Y, Z$ on $V$. This metric is used to measure the size of tensors. For any tensorfield $\Phi$ we define the supremum norms by

$$
\begin{gathered}
\|\Phi(x)\|=\bar{g}_{E}(\Phi(x), \Phi(x))^{1 / 2}, \quad x \in V \\
\|\Phi\|=\sup \{\|\Phi(x)\|, x \in V\} \\
\|\Phi\|_{k}=\sum_{j=0}^{k}\left\|\bar{\nabla}^{j} \Phi\right\| .
\end{gathered}
$$


The Riemannian geodesic distance with respect to $\bar{g}_{E}$ we denote by $d$.

Throughout this paper, we will assume the timelike convergence condition

$$
\overline{\operatorname{Ric}}(Y, Y) \geq 0
$$

for timelike vectorfields $Y$ although this can be weakened in many cases to the condition

$$
\overline{\operatorname{Ric}}(Y, Y) \geq \kappa\langle Y, Y\rangle
$$

for timelike vectorfields $Y$ and some constant $\kappa \geq 0$ (see [E1]). If $\bar{g}$ satisfies the Einstein equations condition $(\kappa)$ holds as a consequence of the weak energy conditions with $\kappa$ depending on the cosmological constant and a bound for the scalar curvature.

A time function $t \in C^{1}(U), U \subset V$ has everywhere past-directed timelike vectorfield $\bar{\nabla} t$. The level sets of $t$ are then spacelike hypersurfaces, that is they admit a timelike normal vector at every point. Their future-directed unit normal vector is given by

$$
-\psi \bar{\nabla} t
$$

where the lapse function $\psi$ is defined by

$$
\psi^{-2}=|\bar{\nabla} t|^{2} \equiv-\langle\bar{\nabla} t, \bar{\nabla} t\rangle .
$$

Using the integral curves of $-\psi^{2} \bar{\nabla} t$ to define (zero-shift) coordinates $(x, t)$ on $U$ the metric $\bar{g}$ takes the form

$$
-\psi^{2}(x, t) d t^{2}+\bar{g}_{i j}(x, t) d x^{i} d x^{j} \quad 1 \leq i, j \leq n .
$$

For a smooth, spacelike hypersurface $M \subset V$ we let $\nu$ be the futuredirected unit normal and

$$
v=-\langle\nu, T\rangle
$$

its gradient function with respect to $T$. The restriction to $T M$ of any tensor field $\Phi$ of order $m$ on $V$ can be estimated by

$$
\left\|\Phi_{\mid T M}(x)\right\| \leq v^{m}(x)\|\Phi(x)\| .
$$

The induced connection and Laplacian on $M$ we denote by $\nabla$ and $\Delta$ respectively. We will sometimes consider

$$
v_{t}=\langle\nu, \psi \bar{\nabla} t\rangle
$$


the gradient function of $M$ with respect to a given time function $t$. Note in particular, that the identity

$$
|\nabla t|^{2}=\psi^{-2}\left(v_{t}^{2}-1\right)
$$

holds (see [B1, (2.6)]). Finally, we define the second fundamental form $A$ of $M$ by $A(Y, Z)=\left\langle\bar{\nabla}_{Y} \nu, Z\right\rangle$ for $Y, Z$ tangent to $M$ and its mean curvature by $H=\operatorname{trace} A$.

Let now $X_{s}=X(\cdot, s): M^{n} \rightarrow V, s \in\left[0, s_{0}\right)$ with $M_{s}=X_{s}\left(M^{n}\right) \subset V$ be a family of spacelike hypersurface embeddings which satisfies

$$
\frac{\partial X}{\partial s}(p, s)=H \nu(p, s)
$$

for $(p, s) \in M^{n} \times\left(0, s_{0}\right)$ where $H$ and $\nu$ are the mean curvature and future directed normal of $M_{s}$. On each $M_{s}$ we adopt the above notation for $M$ usually without the subscript $s$.

With respect to a time function $t$ we will often write the hypersurfaces $M_{s}$ as local graphs of functions $u_{s}=u(\cdot, s)$ over some subset $\Omega \subset \mathcal{S}_{0}=$ $\{x \in V, t(x)=0\}$ using zero shift coordinates. Note that since (MCF) propagates the hypersurfaces $M_{s}$ in the direction of their normal $\nu$ while they are graphs with respect to the integral curves of $-\psi^{2} \bar{\nabla} t$, the local height function $u_{s}$ agrees with $t_{\left.\right|_{M_{s}}}$, the restriction of $t$ to $M_{s}$ only up to a tangential diffeomorphism (depending on $s$ ) in $\Omega$. A calculation using (MCF) shows that

$$
\frac{\partial u}{\partial s}=\psi^{-1} v_{t}^{-1} H
$$

while the time function $t$ restricted to $M_{s}$ satisfies

$$
\frac{\partial t}{\partial s}=\psi^{-1} v_{t} H
$$

Since $v_{t} \geq 1$, equation (1.3) leads to the inequality

$$
\left|\frac{\partial u}{\partial s}\right| \leq \psi^{-1} H
$$

Note also that (1.3) is a quasilinear parabolic equation of the form

$$
\frac{\partial u}{\partial s}=\psi^{-1} \sqrt{1-|\psi D u|^{2}} \operatorname{div}\left(\frac{\psi D u}{\sqrt{1-|\psi D u|^{2}}}\right)+\frac{a\left(\psi, \bar{\nabla} \psi, A_{\mathcal{S}_{0}}, D u\right)}{1-|\psi D u|^{2}}
$$


where $D$, div and $A_{\mathcal{S}_{0}}$ denote the tangential gradient, divergence and second fundamental form on $\mathcal{S}_{0}$ and $a$ is bounded (compare [B1, 2.17]).

We refer to [EH1] for a derivation of the following evolution equations from $(\mathrm{MCF})$.

Proposition 1.1 ([EH1]). Any smooth solution of (MCF) satisfies

$$
\begin{aligned}
\left(\frac{d}{d s}-\Delta\right) t & =-\operatorname{div}_{M_{s}} \bar{\nabla} t \\
\left(\frac{d}{d s}-\Delta\right) v & =-v\left(|A|^{2}+\overline{\operatorname{Ric}}(\nu, \nu)\right)-T\left(H_{T}\right)-H\left\langle\bar{\nabla}_{\nu} T, \nu\right\rangle \\
\left(\frac{d}{d s}-\Delta\right) H & =-H\left(|A|^{2}+\overline{\operatorname{Ric}}(\nu, \nu)\right)
\end{aligned}
$$

where $T\left(H_{T}\right)$ denotes the variation of $H$ with respect to a deformation of $V$ generated by $T$ (see [B1]).

Using the timelike convergence condition one derives from Proposition 1.1 the two main evolution inequalities (Lemma 1.3 and 1.4) used in the proof of the interior estimate of Section 2. Lemma 1.3 also relies on the following consequence of Schwarz' inequality.

Proposition 1.2 ([B1]). On smooth spacelike hypersurfaces the inequality

$$
|A|^{2} v^{2} \geq\left(1+\frac{1}{n}-\epsilon\right)|\nabla v|^{2}-H^{2} v^{2}-C_{\epsilon} v^{4}
$$

holds for any $\epsilon>0$ where $C_{\epsilon}$ depends on $\epsilon^{-1}, n$ and $\|\bar{\nabla} T\|$.

Proposition 1.2 appears in some form in all proofs of gradient type estimates (see $[\mathrm{B} 1,2],[\mathrm{CY}],[\mathrm{G}]$ ).

Lemma 1.3 ([EH1], [B1, B2]). Suppose the timelike convergence condition holds. Then any smooth solution of (MCF) satisfies the inequality

$$
\left(\frac{d}{d s}-\Delta\right) v^{2} \leq-4\left(1+\frac{1}{4 n}\right)|\nabla v|^{2}+C v^{4}+2 H^{2} v^{2}
$$

where $C$ depends on $n$ and $\|T\|_{2}$.

Proof. We repeat the argument in [B2] or [EH1] for the convenience of the reader. Using $[\mathrm{B} 1,(2.10)]$ we estimate the expression $T\left(H_{T}\right)$ in the evolution equation (1.8) by

$$
\left|T\left(H_{T}\right)\right| \leq C\left(v^{3}+v^{2}|A|\right) .
$$


Here $C$ depends on $\left\|\mathcal{L}_{T} \bar{g}\right\|_{1}$ where $\mathcal{L}_{T} \bar{g}$ is the Lie derivative of the metric with respect to $T$ whose $C^{1}$ - norm can be controlled in terms of $\|T\|_{2}$. By $(1.1)$

$$
\left|\left\langle\bar{\nabla}_{\nu} T, \nu\right\rangle\right| \leq v^{2}\|\bar{\nabla} T\|
$$

In view of the timelike convergence condition, equation (1.8) then leads to

$$
\left(\frac{d}{d s}-\Delta\right) v^{2} \leq-2 v^{2}|A|^{2}+C\left(v^{4}+v^{3}|A|\right)-2|\nabla v|^{2}
$$

where $C$ depends on $n$ and $\|T\|_{2}$. Inequality (1.11) is now a consequence of (1.10), Young's inequality $a b \leq \epsilon a^{2}+\frac{1}{4 \epsilon} b^{2}$ applied to the $v^{3}|A|$ - term and an appropriate choice of $\epsilon$ depending on $n$.

If we use the inequality $|A|^{2} \geq \frac{1}{n} H^{2}$ in combination with the timelike convergence condition, the evolution equation (1.9) implies

Lemma 1.4 ([EH1]). Suppose the timelike convergence condition holds. Then any smooth solution of (MCF) satisfies the inequality

$$
\left(\frac{d}{d s}-\Delta\right) H^{2} \leq-\frac{2}{n} H^{4}
$$

Note that without the timelike convergence condition the form of (1.11) does not change except that $C$ will now also depend on $\|\overline{\mathrm{Ric}}\|$. However, in (1.12) we would obtain an additional term of the form $2\|\overline{\operatorname{Ric}}\| v^{2} H^{2}$ on the right hand side which we cannot handle in the a priori estimates of Section 2.

When the hypersurfaces $M_{s}$ are compact without boundary (as in the case of cosmological spacetimes considered in [EH1]) or solve an initialboundary value problem with $\partial M_{s}=\partial M_{0}$ (see section 3$)$ the parabolic maximum principle can be applied to obtain height estimates. The maximum principle also holds in some noncompact situations (for conditions see for example [EH2, Thm 4.3] or [E1, Thm 1.2]). In these situations the mean curvature and local height functions of the solution can be controlled (compare also [EH1] and $[\mathrm{E} 1,2])$.

Proposition 1.5. Let $\left(M_{s}\right)_{s \in\left(0, s_{0}\right)}$ be a smooth solution of $(M C F)$ with smooth, spacelike initial data $M_{0}$. Suppose that this solution is compact either without boundary or smooth up to the boundary satisfying $\partial M_{s}=\partial M_{0}$ for all $s \in\left(0, s_{0}\right)$ or that it is noncompact but satisfies the conditions of the 
noncompact maximum principle. Assume also that the timelike convergence condition holds. Then the mean curvature satisfies the inequality

$$
\sup _{M_{s}} H^{2} \leq \frac{n}{2 s}
$$

for all $s \in\left(0, s_{0}\right)$. If furthermore the hypersurfaces $M_{s}$ can be written as local graphs of functions $u_{s}$ over some open set $\Omega \subset \mathcal{S}_{0}=\{t=0\}$ where $t$ is a given time function with lapse $\psi$, then the inequality

$$
\left|u_{s}(x)-u_{s^{\prime}}(x)\right| \leq \frac{1}{\psi(x)} \sqrt{2 n\left|s-s^{\prime}\right|}
$$

holds for all $x \in \Omega$ and $s, s^{\prime} \in\left[0, s_{0}\right)$.

Proof. From Lemma 1.4. we calculate

$$
\left(\frac{d}{d s}-\Delta\right) s H^{2} \leq-\frac{2}{n} s H^{4}+H^{2} .
$$

By the compact maximum principle, $s H^{2}$ can never reach a maximum larger than $n / 2$. Note that in the case where the hypersurfaces are smooth up to the boundary and satisfy $\partial M_{s}=\partial M_{0}$ we are using that $\frac{\partial t}{\partial s}=0$ and therefore $H=0$ on $\partial M_{s}$ for all $s \in\left(0, s_{0}\right)$ in view of (1.4). Here $H$ is the extension of the mean curvature of $M_{s}$ to $\partial M_{s}$.

In the noncompact case, we apply the noncompact maximum principle of [EH2] to the function $\max \left(s H^{2}-\frac{n}{2}, 0\right)$ (see [E1, Cor. 1.4]).

Inequality (1.16) is now obtained by combining (1.5) and (1.15) followed by a pointwise integration with respect to the $s$ variable.

Let us more generally consider solutions of

$$
\frac{\partial X}{\partial s}=(H-\mathcal{H}) \nu
$$

where we additionally assume the condition

$$
Y(\mathcal{H}) \geq 0
$$

for all future directed timelike vectorfields $Y$ as in [EH1]. If we also relax the timelike convergence condition to condition $(\kappa)$ then inequalities (1.11) and (1.12) become

$$
\left(\frac{d}{d s}-\Delta\right) v^{2} \leq-4\left(1+\frac{1}{4 n}\right)|\nabla v|^{2}+C v^{4}+4(H-\mathcal{H})^{2} v^{2}
$$


and

$$
\left(\frac{d}{d s}-\Delta\right)(H-\mathcal{H})^{2} \leq-\frac{1}{n}(H-\mathcal{H})^{4}+C
$$

where now $C$ depends additionally on $\kappa$ and $\|\mathcal{H}\|_{1}$ in (1.17) and $\kappa$ and $\|\mathcal{H}\|$ in (1.18) (see [E1]).

For a statement of the evolution equations for the second fundamental form and its covariant derivatives we refer to [EH1] or [E1]. Once estimates for $v$ have been established bounds on these quantities replace standard parabolic Schauder estimates for second and higher derivatives of solutions of equation (1.6) (see Theorem 2.2 below).

\section{Interior Estimates.}

In this section we will assume that $\tau \in C^{2}(V)$ is a time function in the region $\{\tau>0\}$ such that

$$
M_{s} \cap\{\tau \geq 0\} \text { is compact }
$$

and

$$
\partial M_{s} \cap\{\tau>0\}=\emptyset
$$

for all $s \in\left[0, s_{0}\right]$ and that there are constants $C_{0}, C_{1}$ and $C_{2}$ such that

$$
\begin{aligned}
\langle\bar{\nabla} \tau, \bar{\nabla} \tau\rangle & \leq-C_{0}^{-2} \\
\|\tau\|_{2} & \leq C_{1} \\
\|T\|_{2} & \leq C_{2}
\end{aligned}
$$

where the norms are taken over the set $\bigcup_{s \in\left[0, s_{0}\right]} M_{s} \cap\{\tau \geq 0\}$.

In [B2, section 3], time functions were constructed which satisfy these conditions with respect to a fixed spacelike hypersurface, see also Section 3 of this paper for a statement of the construction. Given an estimate on the height of the hypersurfaces $M_{s}$ above $M_{0}$ one can usually determine an interval $\left[0, s_{0}\right]$ such that a timefunction constructed with respect to $M_{0}$ also satisfies the conditions for $M_{s}$ when $s \in\left[0, s_{0}\right]$. Such height estimates generally follow from a maximum principle as in Proposition 1.5. In the initial - boundary value problems considered in Section 3, the hypersurfaces $M_{s}$ satisfy the above conditions for all $s>0$ as they are contained in the domain of dependence of $M_{0}$.

There are, however, solutions of (MCF) to which the maximum principle does not apply (see [E2]) such as the translating solution in Minkowski space given by the function $u_{s}(x)=\log \cosh x+s$ which has exponentially growing 
curvature at infinity and is geodesically incomplete. Here the graph of the function $u_{s}$ lies initially underneath the homothetic solution of (MCF) given by $\left(x^{2}+2 s\right)^{1 / 2}$ but crosses it at infinity for $s=\log 2$. Since for such solutions $H^{2}$ is large on $M_{0} \cap\{\tau \geq 0\}$, the above conditions will usually be satisfied only for small $s_{0}$ as $M_{s} \cap\{\tau \geq 0\}$ becomes noncompact very quickly (see the discussion in the appendix.)

Theorem 2.1. Let $\left(M_{s}\right)_{s \in\left(0, s_{0}\right]}$ be a smooth solution of $(M C F)$ with smooth, spacelike initial data $M_{0}$. Let $\tau \in C^{2}(V)$ be a time function satisfying the above conditions. Suppose also that the timelike convergence condition holds. Then for all $s \in\left[0, s_{0}\right]$ the gradient function and the mean curvature satisfy the estimates

$$
\sup _{M_{s} \cap\{\tau \geq 0\}} v^{2}\left(\Lambda-H^{2}\right)^{-1 / q} \tau^{p} \leq C+\sup _{M_{0} \cap\{\tau \geq 0\}} v^{2}\left(\Lambda-H^{2}\right)^{-1 / q} \tau^{p}
$$

for $\Lambda>\max \left\{n / 2, \sup _{M_{0} \cap\{\tau \geq 0\}} H^{2}\right\}$ and

$$
\sup _{M_{s} \cap\{\tau \geq 0\}} s v^{2}\left(\Lambda-s H^{2}\right)^{-1 / q} \tau^{p} \leq C\left(1+s_{0}\right)^{2}
$$

for $\Lambda>n / 2$ where $q=q(n)>0$ and $C, p>0$ depend on $n, C_{0}, C_{1}, C_{2}$ and

$$
\tau_{\max } \equiv \sup _{s \in\left[0, s_{0}\right] M_{s}} \sup _{M_{s}} \tau
$$

as long as $v$ and the quantities involving $H^{2}$ are finite on $M_{s}$ for $s \in\left[0, s_{0}\right]$. The constant $C$ additionally depends on $\Lambda-\max \left\{n / 2, \sup _{M_{0} \cap\{\tau \geq 0\}} H^{2}\right\}$ or $\Lambda-n / 2$. As a consequence of (2.1) and (2.2) the estimate

$$
\sup _{M_{s} \cap\{\tau \geq \alpha\}} v^{2} \leq C_{\alpha}\left(1+\frac{1}{s}\right)
$$

holds for any $\alpha>0$ and $s \in\left(0, s_{0}\right]$ with $C_{\alpha}$ depending on $\alpha^{-1}, n, C_{0}, C_{1}, C_{2}$ and $\tau_{\max }$.

Remarks. (i) The estimates of Theorem 2.1 with $H$ replaced by $H-\mathcal{H}$ also hold for solutions of $(\mathrm{MCF})_{\mathcal{H}}$ as long as $\mathcal{H} \in C^{2}(V)$ satisfies

$$
Y(\mathcal{H}) \geq 0
$$

for all future - directed timelike vectorfields $Y$ (compare [EH1]). The constants will then additionally depend on $\|\mathcal{H}\|_{2}$. The timelike convergence condition can also be weakened to condition $(\kappa)$ discussed in the previous 
section if we additionally impose a restriction on the size of $s_{0}$ or alternatively choose $\Lambda$ large enough depending on $\kappa^{-1}$. The proof of Theorem 2.1 is easily modified to incorporate these more general assumptions using inequalities (1.17) and (1.18) instead of (1.11) and (1.12).

(ii) Recall that in the cases where the maximum principle is applicable to a solution of $(\mathrm{MCF})$ the inequality

$$
\sup _{M_{s}} H^{2} \leq \frac{n}{2 s}
$$

holds for all $s \in\left(0, s_{0}\right]$ by Proposition 1.5. The proof of Theorem 2.1 then simplifies significantly as one only needs to consider the testfunction $s v^{2} \tau^{p}$ in this case, similarly as in [B2, proof of Thm 3.1]). Theorem 2.1 implies (1.15) for $M_{s}$ inside $\{\tau \geq 0\}$ even if the solution does not obey the maximum principle. However, in general $s_{0}$ may be small in these cases so that we cannot let $s \rightarrow \infty$.

(iii) In [E2], an alternative version of (2.1) in Minkowski space $\mathbf{R}^{n, 1}$ was established inside the sets $\left\{(x, t) \in \mathbf{R}^{n, 1},|x|^{2}-t^{2} \leq \rho^{2}\right\}$.

Proof of Theorem 2.1. We will only present the proof of (2.2) by deriving an evolution inequality for the function

$$
f=s v^{2}\left(\Lambda-s H^{2}\right)^{-1 / q} \tau^{p} .
$$

The argument can be adopted almost without change in order to establish estimate (2.1). One simply has to omit the factors $s$ in $f$.

Estimate (2.3) follows by combining (2.1) and (2.2) for a fixed $\Lambda>n / 2$ : If $s_{0} \leq 1,(2.3)$ is an immediate consequence of (2.2). If $s_{0}>1$ we first apply (2.2) for $s \in(0,1]$ and then use (2.1) to estimate $v$ and $H$ for $s \geq 1$ in terms of their bounds at $s=1$.

Recall that we are assuming that $f$ is finite for $s \in\left[0, s_{0}\right]$ and now aim to establish a bound on $f$ which only depends on its initial values. In the following all constants which depend on $n, C_{0}, C_{1}, C_{2}$ and $\tau_{\max }$ will be denoted by $C$.

From (1.7) for the time function $\tau$ and the inequality $\left|\operatorname{div}_{M_{s}} \bar{\nabla} \tau\right| \leq$ $v^{2}\left\|\bar{\nabla}^{2} \tau\right\|$ which follows from (1.1) we obtain

$$
\left|\left(\frac{d}{d s}-\Delta\right) \tau\right| \leq C_{1} v^{2} .
$$

Let $\mathcal{T}=-\frac{\bar{\nabla} \tau}{|\bar{\nabla} \tau|}$ be the future - directed unit normal to the $\tau$-foliation where

$$
|\bar{\nabla} \tau|^{2} \equiv-\langle\bar{\nabla} \tau, \bar{\nabla} \tau\rangle \geq C_{0}^{-2}
$$


by assumption. We then estimate

$$
-\langle\mathcal{T}, T\rangle=\frac{\langle\bar{\nabla} \tau, T\rangle}{|\bar{\nabla} \tau|} \leq C_{0} C_{1}
$$

As in [B2, section 3] we use the identity

$$
|\nabla \tau|^{2}=|\bar{\nabla} \tau|^{2}\left(\langle\nu, \mathcal{T}\rangle^{2}-1\right)
$$

(see (1.2)) and the inequality

$$
v=-\langle\nu, T\rangle \leq 2\langle\mathcal{T}, T\rangle\langle\nu, \mathcal{T}\rangle
$$

for unit future - directed timelike vectors ([B2, Lemma 2.1]) to infer

$$
|\nabla \tau|^{2} \geq C_{0}^{-2}\left(\frac{1}{4 C_{0}^{2} C_{1}^{2}} v^{2}-1\right)
$$

From Lemma 1.4 we obtain

$$
\left(\frac{d}{d s}-\Delta\right) s H^{2} \leq-\frac{2 s}{n} H^{4}+H^{2} .
$$

Similarly to [E2, section 3] we first derive an evolution equation for the function

$$
f=s v^{2} h\left(s H^{2}\right)
$$

where we will substitute $h(r)=(\Lambda-r)^{-1 / q}$ later. Denoting derivatives of $h$ by ', we calculate for $w=s^{-1} f=v^{2} h$ from Lemma 1.3 and (2.7) using also the inequalities $h \geq 0$ and $h^{\prime} \geq 0$

$$
\begin{aligned}
\left(\frac{d}{d s}-\Delta\right) w \leq & -4\left(1+\frac{1}{4 n}\right)|\nabla v|^{2} h+C v^{4} h+2 H^{2} v^{2} h-\frac{2 s}{n} H^{4} v^{2} h^{\prime} \\
& +H^{2} v^{2} h^{\prime}-s^{2}\left|\nabla H^{2}\right|^{2} v^{2} h^{\prime \prime}-2 s\left\langle\nabla v^{2}, \nabla H^{2}\right\rangle h^{\prime} .
\end{aligned}
$$

With the help of Young's inequality $a b \leq \epsilon a^{2}+\frac{1}{4 \epsilon} b^{2}$ for $\epsilon=\frac{1}{2 n}$, we estimate

$$
\left|2 s\left\langle\nabla v^{2}, \nabla H^{2}\right\rangle h^{\prime}\right|=\left|4 s v\left\langle\nabla v, \nabla H^{2}\right\rangle h^{\prime}\right| \leq \frac{1}{2 n}|\nabla v|^{2} h+8 n s^{2} v^{2}\left|\nabla H^{2}\right|^{2} \frac{\left(h^{\prime}\right)^{2}}{h}
$$

and therefore

$$
\begin{aligned}
\left(\frac{d}{d s}-\Delta\right) w \leq & -4\left(1+\frac{1}{8 n}\right)|\nabla v|^{2} h+C v^{4} h+2 H^{2} v^{2} h-\frac{2 s}{n} H^{4} v^{2} h^{\prime} \\
& +H^{2} v^{2} h^{\prime}+s^{2}\left(8 n \frac{\left(h^{\prime}\right)^{2}}{h}-h^{\prime \prime}\right)\left|\nabla H^{2}\right|^{2} v^{2}
\end{aligned}
$$


The inequality $|a+b|^{2} \leq(1+\delta)|a|^{2}+(1+1 / \delta)|b|^{2}$ for $\delta>0$ yields

$|\nabla w|^{2}=\left|2 v \nabla v h+s v^{2} \nabla H^{2} h^{\prime}\right|^{2} \leq 4(1+\delta) v^{2}|\nabla v|^{2} h^{2}+s^{2}\left(1+\delta^{-1}\right) v^{4}\left|\nabla H^{2}\right|^{2}\left(h^{\prime}\right)^{2}$.

This implies

$$
(1+\delta) \frac{|\nabla w|^{2}}{w} \leq 4(1+\delta)^{2}|\nabla v|^{2} h+s^{2}(1+\delta)\left(1+\delta^{-1}\right) v^{2}\left|\nabla H^{2}\right|^{2} \frac{\left(h^{\prime}\right)^{2}}{h}
$$

We then choose $\delta=\delta(n)>0$ such that $(1+\delta)^{2}=1+\frac{1}{8 n}$ and substitute (2.9) into (2.8) to arrive at

$$
\begin{aligned}
\left(\frac{d}{d s}-\Delta\right) w \leq & -(1+\delta) \frac{|\nabla w|^{2}}{w}+C v^{4} h+2 H^{2} v^{2} h-\frac{2 s}{n} H^{4} v^{2} h^{\prime} \\
& +H^{2} v^{2} h^{\prime}+s^{2}\left(c(n) \frac{\left(h^{\prime}\right)^{2}}{h}-h^{\prime \prime}\right)\left|\nabla H^{2}\right|^{2} v^{2} .
\end{aligned}
$$

The function $h$ satisfies

$$
\left(c(n) \frac{\left(h^{\prime}\right)^{2}}{h}-h^{\prime \prime}\right)(r)=\frac{1}{q^{2}}(c(n)-q-1)(\Lambda-r)^{-\frac{1}{q}-2} \leq 0
$$

if $q+1 \geq c(n)$. We may therefore discard the $\left|\nabla H^{2}\right|^{2}-$ term on the right hand side. For $f=s w=s v^{2} h$ this leads to

$$
\begin{aligned}
\left(\frac{d}{d s}-\Delta\right) f \leq & -(1+\delta) \frac{|\nabla f|^{2}}{f}+C v^{2} f+2 H^{2} f \\
& -\frac{2 s}{n} H^{4} \frac{h^{\prime}}{h} f+H^{2} \frac{h^{\prime}}{h} f+s^{-1} f .
\end{aligned}
$$

Multiplying by $\tau^{p}$ for $p>0$ we calculate inside the set $\{\tau>0\}$

$$
\begin{aligned}
\left(\frac{d}{d s}-\Delta\right) f \tau^{p} \leq & -(1+\delta) \frac{|\nabla f|^{2}}{f} \tau^{p}+C v^{2} f \tau^{p}+2 H^{2} f \tau^{p} \\
& -\frac{2 s}{n} H^{4} \frac{h^{\prime}}{h} f \tau^{p}+H^{2} \frac{h^{\prime}}{h} f \tau^{p}+s^{-1} f \tau^{p} \\
& +p f \tau^{p-1}\left|\left(\frac{d}{d s}-\Delta\right) \tau\right|-p(p-1) \tau^{p-2}|\nabla \tau|^{2} f \\
& -2 p \tau^{p-1}\langle\nabla f, \nabla \tau\rangle .
\end{aligned}
$$

We now estimate

$$
\left|2 p \tau^{p-1}\langle\nabla f, \nabla \tau\rangle\right| \leq(1+\delta) \frac{|\nabla f|^{2}}{f} \tau^{p}+\frac{p^{2}}{1+\delta} \tau^{p-2}|\nabla \tau|^{2} f
$$


use (2.4) and choose $p=p(n)$ so large that $-\frac{\delta}{1+\delta} p^{2}+p \leq-\frac{\delta}{2(1+\delta)} p^{2}$. This yields

$$
\begin{aligned}
\frac{1}{f \tau^{p}}\left(\frac{d}{d s}-\Delta\right) f \tau^{p} \leq & C v^{2}+2 H^{2}-\frac{2 s}{n} H^{4} \frac{h^{\prime}}{h}+H^{2} \frac{h^{\prime}}{h} \\
& +s^{-1}+p C v^{2} \tau^{-1}-\frac{\delta}{2(1+\delta)} p^{2} \tau^{-2}|\nabla \tau|^{2} .
\end{aligned}
$$

Using (2.5), we bound $|\nabla \tau|^{2}$ from below in terms of $v^{2}$ and then make $p=p\left(n, C, \tau_{\max }\right)$ even larger to achieve

$$
\frac{1}{f \tau^{p}}\left(\frac{d}{d s}-\Delta\right) f \tau^{p} \leq-\tau^{-2} v^{2}-\frac{2 s}{n} H^{4} \frac{h^{\prime}}{h}+\left(2+\frac{h^{\prime}}{h}\right) H^{2}+s^{-1}+C \tau^{-2} .
$$

At a point in $M_{s_{1}} \cap\{\tau \geq 0\}$ for $s_{1} \in\left(0, s_{0}\right]$ where $f \tau^{p}$ takes its largest maximum on any $M_{s}$ over the interval $\left[0, s_{0}\right]$ we have

$$
\left(\frac{d}{d s}-\Delta\right) f \tau^{p} \geq 0
$$

Here we have used the compactness of $M_{s} \cap\{\tau \geq 0\}$. In view of (2.10) we obtain at this maximum point after substituting $\frac{h^{\prime}}{h}=\frac{1}{q}\left(\Lambda-s_{1} H^{2}\right)^{-1}$ and multiplying by $s_{1}$

$$
\tau^{-2} s_{1} v^{2}+\frac{2}{n q} \frac{s_{1}^{2} H^{4}}{\Lambda-s_{1} H^{2}} \leq C\left(1+s_{1} \tau^{-2}\right)+\left(2+\frac{1}{q\left(\Lambda-s_{1} H^{2}\right)}\right) s_{1} H^{2} .
$$

We now set $\theta \equiv \Lambda-\frac{n}{2}>0$.

Case 1. Suppose that at the maximum point the inequality

$$
s_{1} H^{2} \geq \Lambda-\gamma \geq \frac{n+\theta}{2}
$$

holds for some $\gamma \in(0, \theta / 2)$ to be chosen. Since $q>1$ this implies

$$
s_{1} H^{2} \geq q \gamma^{1-\frac{1}{q}} \geq q\left(\Lambda-s_{1} H^{2}\right)^{1-\frac{1}{q}}
$$

for sufficiently small $\gamma$ depending on $q=q(n)$ and hence

$$
\frac{s_{1} H^{2}}{q\left(\Lambda-s_{1} H^{2}\right)} \geq h \text {. }
$$

Furthermore, again for sufficiently small $\gamma$ depending on $n$ and $\theta$ we have

$$
\frac{s_{1} H^{2}}{q\left(\Lambda-s_{1} H^{2}\right)} \geq \frac{n+\theta}{2 q \gamma} \geq \frac{4 n}{\theta} .
$$


Combining (2.12) - (2.14) we obtain

$$
\frac{2}{n q} \frac{s_{1}^{2} H^{4}}{\Lambda-s_{1} H^{2}} \geq \frac{\theta}{2 n} h+\left(2+\frac{1}{q\left(\Lambda-s_{1} H^{2}\right)}\right) s_{1} H^{2} .
$$

Substituting this into (2.11) and multiplying by $\tau^{2}$ yields

$$
s_{1} v^{2}+\frac{\theta}{2 n} h \tau^{2} \leq C\left(1+s_{1}\right)
$$

and therefore

$$
s_{1} v^{2} h \tau^{p} \leq C\left(1+s_{1}\right)^{2} \tau^{p-2}
$$

where now $C$ depends also on $\theta^{-1}$. Since $s_{1} \leq s_{0}$ this implies

$$
f \leq C\left(1+s_{0}\right)^{2}
$$

at the maximum of $f$ on $\left[0, s_{0}\right]$ in this case.

Case 2. If $s_{1} H^{2}<\Lambda-\gamma$ at the maximum point where $\gamma=\gamma(n, \theta) \in$ $(0, \theta / 2)$ was chosen in case 1 we estimate the $\left(\Lambda-s_{1} H^{2}\right)$ - expressions on the right hand side of (2.11) in terms of $\gamma^{-1}$, discard the $H^{4}$ - term on the left hand side and multiply by $\tau^{2}$ to obtain as before

$$
s_{1} v^{2} \leq C\left(1+s_{0}\right) .
$$

In view of the inequality $h<\gamma^{-1 / q}$ we arrive at

$$
f \leq C\left(1+s_{0}\right)
$$

in this case. Combining the two cases, we conclude that for all $s \in\left[0, s_{0}\right]$ the estimate

$$
s v^{2}\left(\Lambda-s H^{2}\right)^{-1 / q} \tau^{p} \leq C\left(1+s_{0}\right)^{2}
$$

holds on $M_{s} \cap\{\tau \geq 0\}$.

Theorem 2.2. Let $\left(M_{s}\right)_{s \in\left(0, s_{0}\right]}$ be a solution of (MCF) satisfying the conditions of Theorem 2.1. Then the second fundamental form of $M_{s}$ and its covariant derivatives satisfy

$$
\sup _{M_{s} \cap\{\tau \geq \alpha\}}\left|\nabla^{m} A\right|^{2} \leq C
$$

for any $\alpha>0, s \in\left(0, s_{0}\right]$ and integer $m \geq 0$ where $C$ depends on $n, m, \alpha^{-1}$, $s^{-1}, C_{0}, C_{1}, C_{2}$ and $\|\overline{\operatorname{Riem}}\|_{m+1}$. 
Proof of Theorem 2.2. For any $\beta \in(0, \alpha)$ and $s_{1} \in\left(0, s_{0}\right)$, estimate $(2.3)$ implies a bound on $v$ in $M_{s} \cap\{\tau \geq \beta\}$ for all $s \in\left[s_{1}, s_{0}\right]$ in terms of $s_{1}^{-1}, \beta^{-1}$ and the other quantities listed in Theorem 2.1. This yields the estimate

$$
|\nabla \tau|+\left|\left(\frac{d}{d s}-\Delta\right) \tau\right| \leq C
$$

in view of (2.4) and the identities and inequalities

$$
\begin{gathered}
|\nabla \tau|^{2}=|\bar{\nabla} \tau|^{2}\left(\langle\nu, \mathcal{T}\rangle^{2}-1\right), \\
\langle\nu, \mathcal{T}\rangle^{2} \leq 4\langle\mathcal{T}, T\rangle^{2} v^{2}, \\
-\langle\mathcal{T}, T\rangle=\frac{\langle\bar{\nabla} \tau, T\rangle}{|\bar{\nabla} \tau|} \leq C_{0} C_{1}
\end{gathered}
$$

listed thereafter. Here $\mathcal{T}$ denotes the future directed normal to the $\tau$ - foliation. The function $\tau$ therefore satisfies condition (17) in [EH2, section 3] required for a distance function $r$ to be used in interior estimates.

The second fundamental form $A$ and its covariant derivatives satisfy the same evolution inequalities as in the case of (MCF) in a Riemannian manifold treated in [EH2] (in fact the inequality for $|A|^{2}$ is even better in the Lorentzian setting in view of the negative $|A|^{4}$ - term (see [E1, Prop.1.1]) which can be used to control the expressions arising from the ambient geometry).

We can therefore follow the argument in [EH2, section 3] exactly to derive the desired estimates inside the set $\{\tau \geq \alpha\}$ for $s \in\left(s_{1}, s_{0}\right]$. One has to replace the cut-off function $(R-r)^{2}$ used there by $(\tau-\beta)^{2}$, use the flow variable $s-s_{1}$ and note that estimates in terms of $R$ become estimates in terms of $\alpha, \beta$ and $\tau_{\max }$. Since $\beta$ and $s_{1}$ were arbitrary this implies the result. $\diamond$

\section{Initial - boundary value problems.}

In this section we solve an initial - boundary value problem for smooth spacelike data (Theorem 3.1) and then combine this result with the interior estimate (2.3) of Theorem 2.1 (which is independent of the initial values of $v$ and $H$ ) to deal with data which contain null curves. The corresponding elliptic Dirichlet problems for hypersurfaces of prescribed mean curvature were treated in $[\mathrm{B} 1,2]$ and $[\mathrm{G}]$.

For the definition of causal relations we refer to [HE] or [B2]. In particular, the domain of dependence and the future (past) domain of influence of a set $S \subset V$ are denoted by $D(S)$ and $I^{ \pm}(S)$. 
A set $S \subset V$ is called achronal if no two points $x, y \in S$ with $x \neq y$ can be joined by a timelike curve.

Theorem 3.1. Let $M_{0}$ be a smooth, spacelike hypersurface with smooth $\partial M_{0}$ and $K \subset V$ be a compact and globally hyperbolic set such that

$$
D\left(M_{0}\right) \subset \subset K
$$

and $M_{0}$ is achronal with respect to $K$. Suppose also that the timelike convergence condition holds. Then there exists a compact, smooth, spacelike solution $\left(M_{s}\right)_{s \in(0, \infty)}$ of $(M C F)$ with initial data $M_{0}$ such that $\partial M_{s}=\partial M_{0}$ and $M_{s} \subset \subset K$ for all $s \in(0, \infty)$. The mean curvature of this solution satisfies the inequality

$$
\sup _{M_{s}} H^{2} \leq \frac{n}{2 s}
$$

for all $s>0$. Furthermore, for any sequence $\left(s_{k}\right) \rightarrow \infty$ there exists a subsequence (denoted again by $\left(s_{k}\right)$ ) such that $M_{s_{k}} \rightarrow M_{\infty}$ where $M_{\infty}$ is a compact, smooth, spacelike hypersurface satisfying $\partial M_{\infty}=\partial M_{0}$ and $H_{M_{\infty}}=0$.

Remarks. (i) The condition on $D\left(M_{0}\right)$ can be replaced by a barrier assumption (see [B1, Thm 4.3]).

(ii) Theorem 3.1 also holds for solutions of $(\mathrm{MCF})_{\mathcal{H}}$ as long as $\mathcal{H}$ satisfies the condition

$$
Y(\mathcal{H}) \geq 0
$$

for all future directed timelike vectorfields $Y$. The decay estimate (3.1) then holds for $H-\mathcal{H}$ instead of $H$ and the limiting hypersurface $M_{\infty}$ has mean curvature $H_{M_{\infty}}=\mathcal{H}$. The condition on $D\left(M_{0}\right)$ can be replaced by an assumption on the existence of barrier hypersurfaces adapted to $\mathcal{H}$ as in [B1] or [EH1].

(iii) Note that there may be several hypersurfaces with prescribed mean curvature $\mathcal{H}$ spanning the same boundary data. For a discussion of a number of conditions which ensure an exponential convergence rate and therefore unique convergence of $M_{s}$ to a limiting hypersurface $M_{\infty}$ with mean curvature $\mathcal{H}$ we refer to [EH1, p.606].

Proof of Theorem 3.1. We proceed similarly as in [B1] and [EH1]. In view of the assumptions on $D\left(M_{0}\right)$ and $K$ we can apply [B1, Prop. 3.2] to construct a time function $t$ with lapse function $\psi$ inside $K$ for which $M_{0} \subset \mathcal{S}_{0}=\{x \in K, t(x)=0\}$. 
We then note that Lemma 2.2 of [B2] is applicable to smooth solutions of (MCF) with $\partial M_{s}=\partial M_{0}$, since $M_{0}$ and $M_{s}$ are homotopic with respect to a timelike vectorfield (as defined there) via the embedding map $X$. Therefore,

$$
M_{s} \subset \overline{D\left(M_{0}\right)} \subset K
$$

for all $s \in(0, \infty)$ so that $\overline{M_{s}}$ is compact by the compactness assumption on $K$ (here $\bar{A}$ denotes the closure of a set with respect to the Riemannian distance $d$ ). In particular, this yields the height estimate

$$
t_{\max } \equiv \sup _{s \in(0, \infty) M_{s}} \sup _{M_{s}}|t|<\infty
$$

Once a uniform bound on the gradient function (with respect to the $t$ foliation)

$$
v_{t}=\langle\nu, \psi \bar{\nabla} t\rangle
$$

on $M_{s}$ has been established, equation (1.6) becomes uniformly parabolic as $v_{t}$ agrees with the quantity $\left(1-\left|\psi D u_{s}\right|^{2}\right)^{-1 / 2}$ for the height function $u_{s}$ of $M_{s}$ up to tangential diffeomorphisms (depending on s) in $\mathcal{S}_{0}$.

We can therefore as in [B1, proof of Thm 4.1] or [G] employ standard theory for such equations ([LUS]) to find a smooth solution of (MCF) for all $s \in(0, \infty)$ in the class of spacelike hypersurfaces which are graphs over a subset of $\mathcal{S}_{0}$.

Inequality (3.1) was stated in Proposition 1.5. Note that the maximum principle and Lemma 1.4 also yield

$$
\sup _{M_{s}} H^{2} \leq \sup _{M_{0}} H^{2}
$$

In view of (3.2) and (3.3) we are now in the same situation as in [EH1, Prop. 4.4] where for a compact solution of (MCF) without boundary an estimate for $v_{t}$ was established. We give an outline of the proof for the convenience of the reader. Following [B1, proof of thm 3.1], we employ a maximum point argument for the function

$$
e^{ \pm \lambda t} v_{t}
$$

on $M_{s}$ : If at $s_{1}>0$ the function $e^{ \pm \lambda t} v_{t}$ first reaches an interior maximum point larger than $\sup _{M_{0}} e^{ \pm \lambda t} v_{t}=\sup _{M_{0}} v_{t}=1$ we have

$$
\left(\frac{d}{d s}-\Delta\right) e^{ \pm \lambda t} v_{t} \geq 0
$$

there. We then combine (1.7), (1.11) (with $T$ replaced by $-\psi \bar{\nabla} t$ ) and (1.2) and choose $\lambda$ suitably large to obtain an estimate of $v_{t}$ in the interior of $M_{s}$ 
for $s \in(0, \infty)$ in terms of $t_{\max }, \sup _{M_{0}} H^{2}$ and $\|\bar{\nabla} t\|_{2}$. For the details we refer to [EH1, Prop. 4.4]. Note that the notation $u$ and $v$ instead of $t_{\left.\right|_{M_{s}}}$ and $v_{t}$ is used there.

If for $s_{1}>0$ the maximum of the function occurs at a point on $\partial M_{s_{1}}=$ $\partial M_{0}$ we have

$$
\eta\left(e^{ \pm \lambda t_{M_{s}}} v\right) \leq 0
$$

there, where $\eta$ is the inner normal to $\partial M_{s_{1}}=\partial M_{0}$ in $M_{s_{1}}$. Since $\frac{\partial t}{\partial s}=0$ on $\partial M_{s}=\partial M_{0}$ for all $s \in\left(0, s_{0}\right)$ we have $H=0$ on $\partial M_{s}$ where $H$ is the extension of the mean curvature of $M_{s}$ to $\partial M_{s}$. The evolution equation (1.7) furthermore yields

$$
\Delta t=\operatorname{div}_{M_{s}} \bar{\nabla} t
$$

on $\partial M_{s}=\partial M_{0}$ where $\Delta=\Delta_{M_{s}}$.

We are now in the same situation as in the elliptic case for zero mean curvature hypersurfaces treated in [B1] and may therefore copy the boundary calculation of [B1, p163] exactly (with $M$ replaced by $M_{s_{1}}$ ). The argument there uses the fact that $t \equiv 0$ on $\partial M_{0}$ to give an estimate of $v_{t}$ on $\partial M_{0}$ in terms of $H_{\partial M_{0}}$, the mean curvature of $\partial M_{0}$. Again, note the difference in notation.

The uniform estimates on $v_{t}$ for all $s \in(0, \infty)$ imply uniform estimates for the second fundamental form of $M_{s}$ and all its derivatives (see [EH1]). Therefore, for any sequence $\left(s_{k}\right) \rightarrow \infty$ there exists a subsequence (again denoted by $\left.\left(s_{k}\right)\right)$ such that $M_{s_{k}} \rightarrow M_{\infty}$ smoothly where $M_{\infty}$ is a smooth, spacelike hypersurface with $\partial M_{\infty}=\partial M_{0}$ which satisfies $H_{M_{\infty}}=0$ in view of (3.1).

Before proceeding to data which contain null curves let us first list those definitions of [B2, Section 3] needed here.

$S \subset V$ is called a weakly spacelike hypersurface if for each $x \in S$ there is a neighbourhood $U$ of $x$ such that $S \cap U$ is an embedded, achronal, $C^{0,1}$ hypersurface which is closed in $U$ (for an equivalent definition see [B2]).

The boundary of a weakly spacelike hypersurface $S$ is defined by $\partial S=$ $\bar{S} \sim S$ where $\bar{S}$ denotes the closure of $S$. Note that this definition allows quite pathological situations as for example $\partial S$ containing isolated points (see [B2] for a discussion).

The singular set of $S$ is defined by

$$
\begin{aligned}
\operatorname{sing} S=\{ & x \in S, x=\gamma\left(l_{0}\right) \text { for some } 0<l_{0}<1, \text { where } \\
& \gamma:[0,1] \rightarrow V \text { is a null geodesic such that } \gamma(l) \in S \\
& \text { for all } l \in(0,1) \text { and } \gamma(0), \gamma(1) \in \partial S\} .
\end{aligned}
$$


Our main theorem states that a solution of (MCF) retains those null geodesics of its initial data which extend to the boundary in both directions but becomes instantaneously smooth and spacelike away from these.

Theorem 3.2. Let $M_{0}$ be a weakly spacelike hypersurface and $K$ be a compact globally hyperbolic set such that

$$
D\left(M_{0}\right) \subset \subset K
$$

and $M_{0}$ is achronal with respect to $K$. Suppose also that the timelike convergence condition holds. Then there exists a family $\left(M_{s}\right)_{s \in(0, \infty)}$ of weakly spacelike hypersurfaces in $K$ with

$$
\partial M_{s}=\partial M_{0} \quad \text { and } \quad \operatorname{sing} M_{s}=\operatorname{sing} M_{0}
$$

for all $s \in(0, \infty)$ such that $\left(M_{s}\right)_{s \in(0, \infty)}$ is a smooth, spacelike solution of (MCF) with initial data $M_{0}$ away from sing $M_{0}$ which satisfies the estimate

$$
\sup _{M_{s} \sim \operatorname{sing} M_{0}} H^{2} \leq \frac{n}{2 s}
$$

for all $s \in(0, \infty)$. Furthermore, for any sequence $\left(s_{k}\right) \rightarrow \infty$ there exists a subsequence (denoted again by $\left(s_{k}\right)$ ) such that $M_{s_{k}} \rightarrow M_{\infty}$ where $M_{\infty}$ is a weakly spacelike hypersurface in $K$ with

$$
\partial M_{\infty}=\partial M_{0} \quad \text { and } \quad \operatorname{sing} M_{\infty}=\operatorname{sing} M_{0}
$$

which is smooth and spacelike in $M_{\infty} \sim \operatorname{sing} M_{0}$ and satisfies $H_{M_{\infty}}=0$ there.

Remark. (i) A corresponding result holds for solutions of $(\mathrm{MCF})_{\mathcal{H}}$.

(ii) The remark after Thm 3.1 regarding convergence of $M_{s}$ to a unique stationary limit $M_{\infty}$ is still relevant here. The arguments of [EH1] carry over to the case where there is a singular set as they only make use of the inequality for $H$ away from the singular set, inequality (1.5) and a pointwise integration argument.

Proof of Theorem 3.2. We proceed similarly as in [B2, Section 4]. In view of the assumptions on $M_{0}$ there is a time function $t$ with lapse $\psi$ inside $K$ such that $M_{0}$ can be written as the graph of a Lipschitz function $u_{0}$ over a bounded subset $\Omega \subset \mathcal{S}_{0}=\{x \in K, t(x)=0\}$. By mollification, we can find a sequence $\left(M_{0}^{j}\right)_{j \in \mathbf{N}}$ of compact, smooth, spacelike hypersurfaces with smooth $\partial M_{0}^{j}$ where each $M_{0}^{j}$ is the graph of a smooth function $u_{0}^{j}$ over a 
domain $\Omega^{j} \subset \Omega$ with smooth $\partial \Omega^{j}$ such that the $M_{0}^{j}$ converge uniformly to $M_{0}$ in the sense that

$$
\sup _{\Omega^{j}}\left|u_{0}^{j}-u_{0}\right| \rightarrow 0
$$

Moreover, the $M_{0}^{j}$ are achronal with respect to $K$ and satisfy $D\left(M_{0}^{j}\right) \subset \subset K$.

By Theorem 3.1, (MCF) admits solutions $\left(M_{s}^{j}\right)_{s \in(0, \infty)}$ of compact, spacelike hypersurfaces with initial data $M_{0}^{j}$ and boundary given by $\partial M_{0}^{j}$ such that $M_{s}^{j} \subset \subset K$ and the mean curvatures of the $M_{s}^{j}$ satisfy the estimate

$$
\sup _{M_{s}^{j}} H^{2} \leq \frac{n}{2 s}
$$

for all $j \in \mathbf{N}$ and $s \in(0, \infty)$. Furthermore, by construction, each $M_{s}^{j}$ is the graph of a smooth function $u_{s}^{j}$ over $\Omega^{j}$.

From Proposition 1.5. we conclude

$$
\sup _{\Omega^{j}}\left|u_{s}^{j}-u_{s^{\prime}}^{j}\right| \leq C \sqrt{2 n\left|s-s^{\prime}\right|}
$$

for all $s, s^{\prime} \in[0, \infty)$ where $C$ depends on a lower bound for $\psi$ in $K$.

In view of this estimate which is uniform in $j$ and the fact that all hypersurfaces are spacelike (which amounts to a uniform Lipschitz condition) the $u_{s}^{j}$ form an equicontinuous sequence over $\mathcal{S}_{0} \times(0, \infty)$. The Arzela-Ascoli Theorem combined with a diagonal sequence argument then implies that a subsequence (again denoted by $\left(M_{s}^{j}\right)$ ) converges uniformly over compact subsets of $\mathcal{S}_{0} \times(0, \infty)$ to a family $\left(M_{s}\right)_{s \in(0, \infty)}$ of weakly spacelike hypersurfaces which satisfy $\partial M_{s}=\partial M_{0}$ for all $s \in(0, \infty)$. Moreover, in view of (3.9) and (3.11) with $s^{\prime}=0$ we conclude that $M_{s} \rightarrow M_{0}$ as $s \rightarrow 0$.

We now fix $s_{0}>0$ and $x_{0} \in M_{s_{0}} \sim\left(\operatorname{sing} M_{0} \cup \partial M_{0}\right)$. Combining the results in [B2, Ch.3] $\left(\left(M_{0}, K\right)\right.$ is a standard data set as defined there) we can find a constant $\alpha>0$, a relatively compact neighbourhood $U$ of $x_{0}$ such that $U \subset \subset K$ and

$$
U \cap\left(\operatorname{sing} M_{0} \cup \partial M_{0}\right)=\emptyset
$$

as well as a function $\tau \in C^{2}(K)$ which is a timefunction in the set $\{\tau \geq 0\}$ and satisfies

$$
U \subset\{\tau \geq \alpha\}
$$

Moreover, the estimates

$$
\begin{aligned}
\langle\bar{\nabla} \tau, \bar{\nabla} \tau\rangle & \leq-C_{0}^{-2} \\
\|\tau\|_{2} & \leq C_{1}
\end{aligned}
$$


hold where $C_{0}$ and $C_{1}$ depend on $M_{0}$ and dist $\left(U\right.$, sing $\left.M_{0} \cup \partial M_{0}\right)$ (distance with respect to $d)$.

In view of (3.9) which implies in particular that

$$
\lim _{j \rightarrow \infty} d_{H}\left(\partial M_{0}^{j}, \partial M_{0}\right)=0
$$

( $d_{H}$ denotes Hausdorff distance with respect to the Riemannian distance function $d$ ) there exists $j_{0} \in \mathbf{N}$ such that for all $j \geq j_{0}$

$$
\partial M_{0}^{j} \cap\{\tau>0\}=\emptyset .
$$

Since $M_{s_{0}}^{j} \rightarrow M_{s_{0}}$ uniformly as $j \rightarrow \infty$ and by (3.11) which is a uniform estimate in $j$ there exists $j_{1} \in \mathbf{N}$ with $j_{1} \geq j_{0}$ and constants $0<s_{1}<s_{0}<$ $s_{2}<\infty$ such that for all $j \geq j_{1}$ and $s \in\left(s_{1}, s_{2}\right)$

$$
M_{s}^{j} \cap U \neq \emptyset .
$$

The sets $M_{s}^{j} \cap\{\tau>0\}$ are compact as $M_{s}^{j} \subset \subset K$. Moreover, we have

$$
\partial M_{s}^{j} \cap\{\tau>0\}=\partial M_{0}^{j} \cap\{\tau>0\}=\emptyset .
$$

Therefore, the conditions of Theorem 2.1 are satisfied with respect to the time function $\tau$. Finiteness of the quantities considered in (2.2) follows from (3.10) and the fact that $v$ is finite on $M_{s}^{j}$. The interior estimates (2.3) and (2.16) applied to $M_{s}^{j} \cap U$ in $\{\tau \geq \alpha\}$ yield

$$
\sup _{M_{s}^{j} \cap U}\left(v+\left|\nabla^{m} A\right|\right) \leq C
$$

for all $x \in M_{s}^{j} \cap U, j \geq j_{1}, s \in\left(s_{1}, s_{2}\right)$ and integers $m \geq 0$ where $C$ depends on $\alpha^{-1}, s_{1}^{-1}$ and the other quantities listed in Theorems 2.1 and 2.2, which in turn depend on the constants in (3.6) and (3.7). Note that $\tau_{\max }$ is bounded since $M_{s}^{j} \subset \subset K$.

The $M_{s}^{j}$ which by (3.12) are uniformly spacelike and smooth in $U$ independently of $j$ therefore converge smoothly for $j \rightarrow \infty$ to $M_{s}$ in $U$ for $s \in\left(s_{1}, s_{2}\right)$. This yields that for all $s_{0}>0$ and every $x_{0} \in M_{s_{0}} \sim$ ( $\left.\operatorname{sing} M_{0} \cup \partial M_{0}\right)$ there is an open set $U \times\left(s_{1}, s_{2}\right)$ containing $\left(x_{0}, s_{0}\right)$ such that $U \cap\left(\operatorname{sing} M_{0} \cup \partial M_{0}\right)=\emptyset$ and $\left(M_{s}\right)_{s \in\left(s_{1}, s_{2}\right)}$ is a smooth, spacelike solution of $(\mathrm{MCF})$ in $U$. As $s_{0}$ and $x_{0}$ were arbitrary we conclude that $\left(M_{s}\right)_{s \in(0, \infty)}$ is a smooth, spacelike solution of (MCF) away from $\operatorname{sing} M_{0} \cup \partial M_{0}$.

Since $\partial M_{s}=\partial M_{0}$ for all $s \in(0, \infty)$ the definition of the singular set implies that

$$
\operatorname{sing} M_{s}=\operatorname{sing} M_{0}
$$


(see [B2, Lemma 3.6]).

To obtain convergence to a mean curvature zero hypersurface we proceed as follows. Every sequence $\left(s_{k}\right) \rightarrow \infty$ has a subsequence (again denoted by $\left.\left(s_{k}\right)\right)$ such that $M_{s_{k}} \rightarrow M_{\infty}$ uniformly where $M_{\infty}$ is a weakly spacelike hypersurface satisfying $\partial M_{\infty}=\partial M_{0}$. Moreover, $\operatorname{sing} M_{\infty}=\operatorname{sing} M_{0}$. To show that $M_{\infty} \sim\left(\operatorname{sing} M_{0} \cup \partial M_{0}\right)$ is smooth and spacelike and satisfies $H=0$ we note that the bounds in (3.12) are independent of $s_{2}$. The sequence $\left(M_{s_{k}}\right)$ therefore is uniformly spacelike and converges smoothly to $M_{\infty}$ away from $\partial M_{0} \cup \operatorname{sing} M_{0}$. Letting $j \rightarrow \infty$ in (3.10) we obtain inequality (3.8) which implies for $\left(s_{k}\right) \rightarrow \infty$ that $H_{M_{\infty}}=0$ in $M_{\infty} \sim\left(\operatorname{sing} M_{0} \cup \partial M_{0}\right)$.

\section{Appendix.}

Let us discuss a simple example along the lines of [B3] involving the time function

$$
\tau(x, t)=\sqrt{t^{2}-|x|^{2}}
$$

in Minkowski space $\mathbf{R}^{n, 1}$.

Let $M_{0}=\operatorname{graph} u_{0} \subset \mathbf{R}^{n, 1}$, where $u_{0}: \Omega \rightarrow \mathbf{R}$ is spacelike that is $\left|u_{0}(x)-u_{0}(y)\right|<|x-y|$ for all $x \neq y \in \Omega \subset \mathbf{R}^{n}$. For $\rho>0$ such that $B_{\rho}(0) \subset \Omega$ we may assume (by vertically translating $u_{0}$ if necessary) that

$$
0<u_{0}(0)=\rho-\sup _{y \in \partial B_{\rho}(0)} u_{0}(y) .
$$

One easily checks that then $u_{0}(x)<|x|-u_{0}(0)$ for $|x| \geq \rho$ and therefore

$$
M_{0} \cap\{\tau \geq 0\} \subset\left\{(x, t) \in \mathbf{R}^{n, 1},|x|<\rho, 0 \leq t<2 \rho\right\}
$$

such that in particular $M_{0} \cap\{\tau \geq 0\}$ is compact.

Let $\left(M_{s}\right)_{s \in\left(0, s_{0}\right)}$ be a solution of (MCF) with initial data $M_{0}$ corresponding to spacelike functions $u_{s}: \Omega \rightarrow \mathbf{R}$. Assume that this solution obeys the parabolic maximum principle (see the discussion in Section 1). Proposition 1.5 (or [E2, Proposition 1.3]) implies

$$
\left|u_{s}(x)-u_{0}(x)\right| \leq \sqrt{2 n s}
$$

for all $x \in \Omega$ and $s \in\left[0, s_{0}\right)$. One then verifies that

$$
M_{s} \cap\{\tau \geq 0\} \subset\left\{(x, t) \in \mathbf{R}^{n, 1},|x|<\rho, 0 \leq t<3 \rho\right\}
$$

and hence $M_{s} \cap\{\tau \geq 0\}$ is compact for all $s \in\left(0, s_{0}\right)$ as long as $\sqrt{2 n s_{0}} \leq$ $u_{0}(0)$. 
Note that in general the maximum (flow) time $s_{0}$ for which the conditions of Theorem 2.1 hold will be finite. The solution $M_{s}$ given by $u_{s}(x)=\log \cosh x+s, x \in \mathbf{R}$ does not intersect $\{\tau>0\}$ at all if $s=0$ but for $s \geq \log 2$

$$
M_{s} \subset\{\tau \geq 0\}
$$

and therefore in particular $M_{s} \cap\{\tau \geq 0\}$ is noncompact.

To determine the constants $C_{0}$ and $C_{1}$ we calculate $\bar{\nabla} \tau(x, t)=-\tau^{-1}(x, t)$ which implies $\langle\bar{\nabla} \tau, \bar{\nabla} \tau\rangle=-1$ and

$$
-\left\langle\bar{\nabla} \tau, \partial_{t}\right\rangle=\frac{t}{\tau}
$$

which is the quantity controlling norms of tensors with respect to $T=\partial_{t}$. The second derivatives of $\tau$ enter the constants of Theorem 2.1 only when estimating the expression $\left(\frac{d}{d s}-\Delta\right) \tau$ (compare (2.14) above). In Minkowski space we have

$$
\left(\frac{d}{d s}-\Delta\right) \tau=\tau^{-1}\left(n+|\nabla \tau|^{2}\right)
$$

analogously to [B3] which can be estimated independently of $\bar{\nabla}^{2} \tau$.

Hence for the time functions $\tau_{\epsilon}=\tau-\epsilon$ we find that $C_{0}=1$ and $C_{1}$ depends on $\epsilon^{-1}$ and the quotient $\epsilon^{-1} \rho$. Note that for $\epsilon=\frac{u_{0}(0)}{2}$ the constant $C_{1}$ is therefore controlled by the reciprocal of the 'gap' quantity

$$
1-\sup _{y \in \partial B_{\rho}(0)} \frac{u_{0}(y)-u_{0}(0)}{\rho} .
$$

\section{References.}

[B1] R. Bartnik, Existence of maximal surfaces in asymptotically flat spacetimes, Comm. Math. Phys. 94, 155-175 (1984)

[B2] R. Bartnik, Regularity of variational maximal surfaces, Acta Math. 166, 145-181 (1988)

[B3] R. Bartnik, Maximal surfaces and general relativity, Proc. miniconf. on Analysis and Geometry, ANU 1986, J. Hutchinson, ed. CMA $(\mathrm{ANU})$

[BS] R. Bartnik and L. Simon, Spacelike hypersurfaces with prescribed boundary values and mean curvature, Comm. Math. Phys 87, 131$152(1982)$ 
[CY] S. Y. Cheng and S. T. Yau, Maximal space-like hypersurfaces in the Lorentz-Minkowski spaces, Annals of Math. 104 (1976), 407-419

[CBY] Y. Choquet-Bruhat and J. York, The Cauchy problem, General Relativity and Gravitation, Vol.I, ed. A.Held, Plenum Press 1980

[E1] K. Ecker, On mean curvature flow of spacelike hypersurfaces in asymptotically flat spacetimes, J. Austral. Math. Soc. (Series A) 55, 41-59 (1993)

[E2] K. Ecker, Interior estimates and longtime solutions for mean curvature flow of noncompact spacelike hypersurfaces in Minkowski space, J. Diff. Geom. 46, 481-498 (1997)

[EH1] K. Ecker and G. Huisken, Parabolic methods for the construction of spacelike slices of prescribed mean curvature in cosmological spacetimes, Comm. Math. Phys. 135, 595-613 (1991)

[EH2] K. Ecker and G. Huisken, Interior estimates for hypersurfaces moving by mean curvature, Invent.math. 105, 547-569 (1991)

[G] C. Gerhardt, H-surfaces in Lorentzian manifolds, Comm. Math. Phys. 89, 523-553 (1983)

[HE] S. W. Hawking and G. F. R. Ellis, The large scale structure of spacetime, Cambridge University Press 1973

[H] G. Huisken, Flow by mean curvature of convex surfaces into spheres, J. Diff. Geom. 20, 237-266 (1984)

[LSU] O. A. Ladyzhenskaya, V. A. Solonnikov and N. N. Uraltseva, Linear and quasilinear equations of parabolic type, Transl. II Ser., Am. Math. Soc. 23 (1968)

[MT] J. E. Marsden and F. J. Tipler, Maximal hypersurfaces and foliations of constant mean curvature in general relativity, Phys. Rep. 66 (3) (1980), 109-139

[SY] R. Schoen and S. T. Yau, Proof of the positive mass theorem I, Comm. Math. Phys. 65, 45-76 (1979)

Department of Mathematics and Statistics, Monash University

Clayton, Vic 3168, Australia

Received March 8, 1999. 